\title{
Grandir en harmonie: Les questions/réponses les plus fréquentes posées par les adolescent(e)s
}

Population Council

Follow this and additional works at: https://knowledgecommons.popcouncil.org/departments_sbsr-rh

Part of the Community Health and Preventive Medicine Commons, Demography, Population, and Ecology Commons, Family, Life Course, and Society Commons, Health Communication Commons, International and Intercultural Communication Commons, and the Medicine and Health Commons How does access to this work benefit you? Let us know!

\section{Recommended Citation}

"Grandir en harmonie: Les questions/réponses les plus fréquentes posées par les adolescent(e)s." Dakar: Population Council, 2007. 


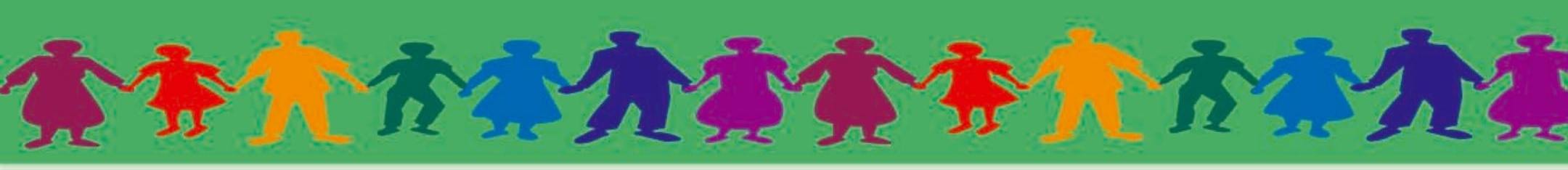

\section{crandir en harmonie}

\section{Les questions/réponses}

Les plus fréquentes posées par les adolescent(e)s
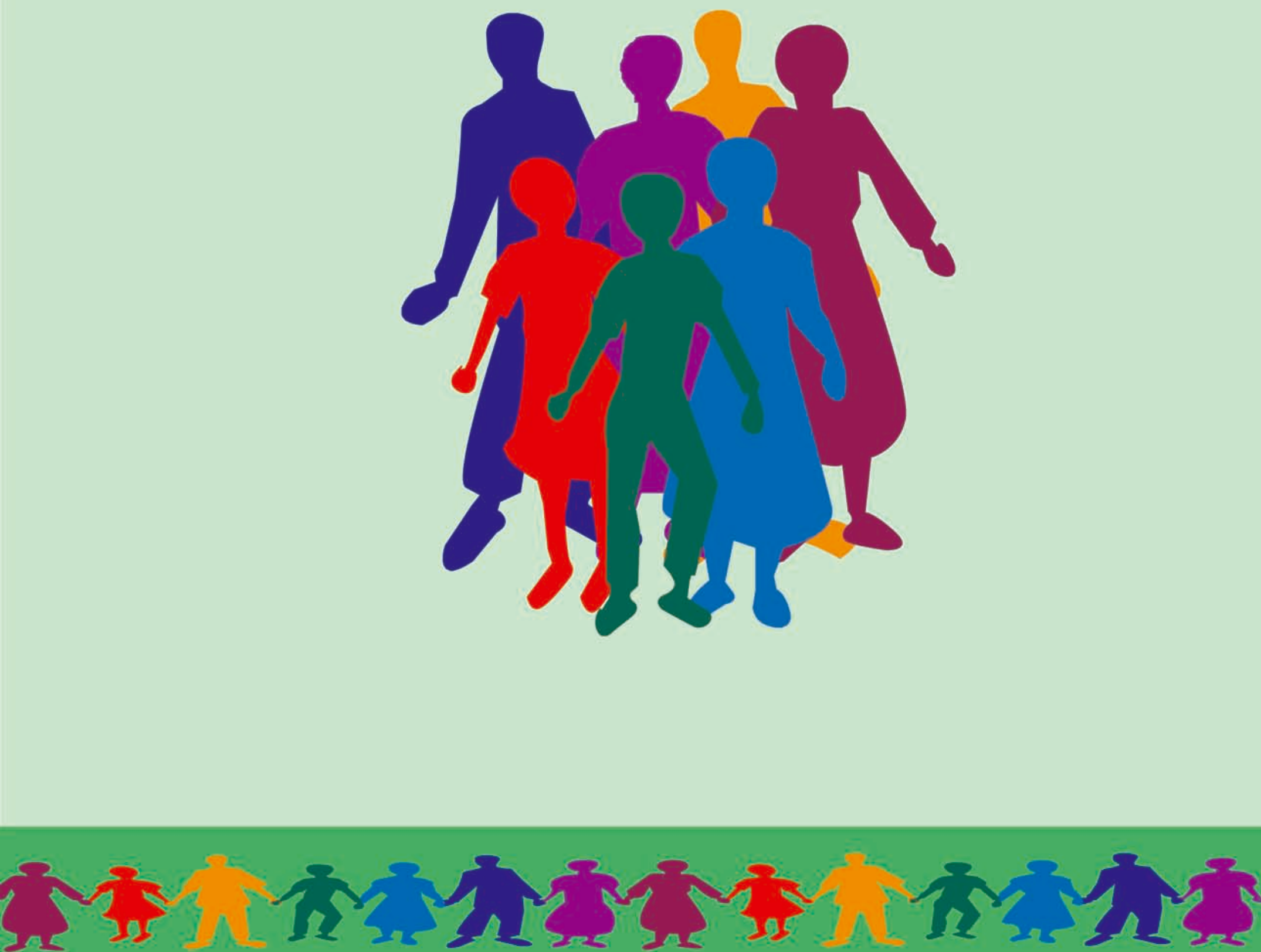


\section{Sommaire}

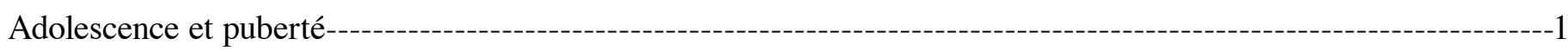

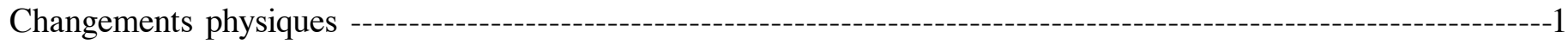

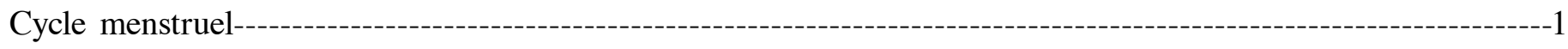

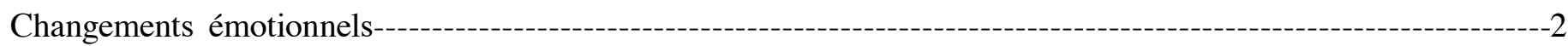

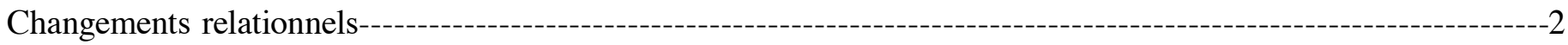

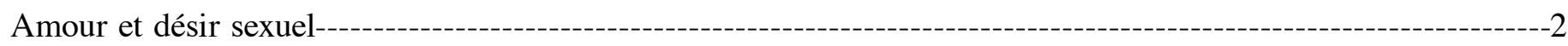

Culture et sexualité------------------------------------------------------------------------------------------------------------------2

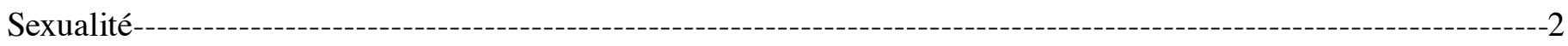

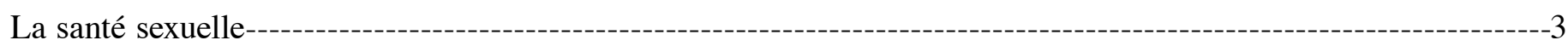

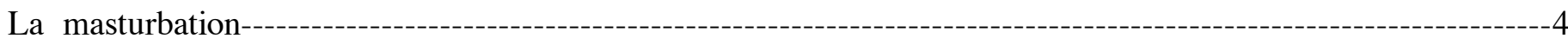

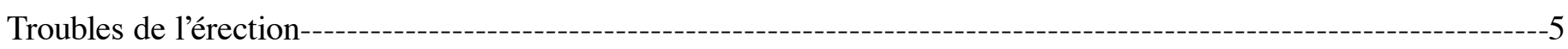

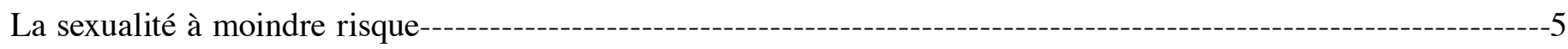

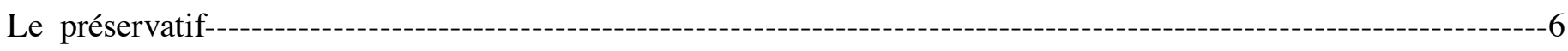

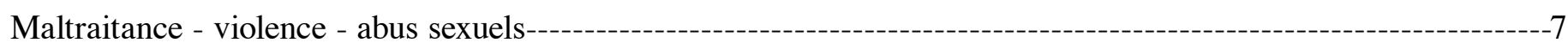

L'homosexualité------------------------------------------------------------------------------------------------------------------7

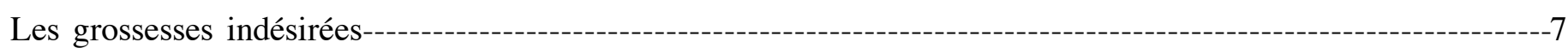

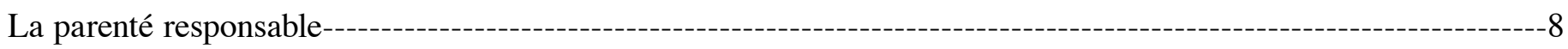

La contraception------------------------------------------------------------------------------------------------------8

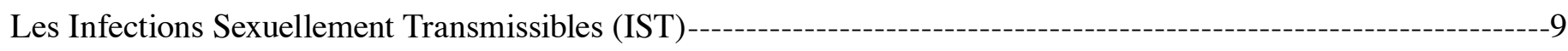

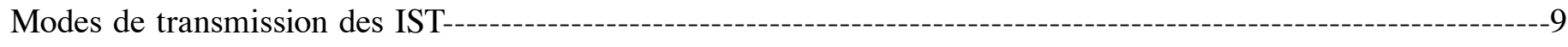

Signes ou symptômes des IST chez la femme et chez l'homme---

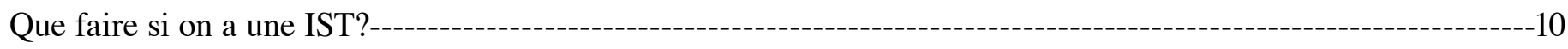

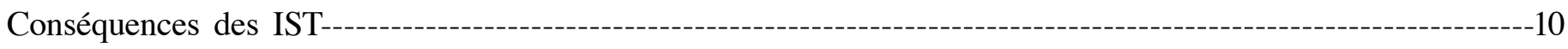

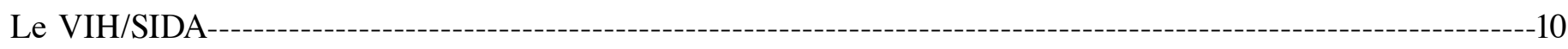

Le test de dépistage------------------------------------------------------------------------------------------------------11

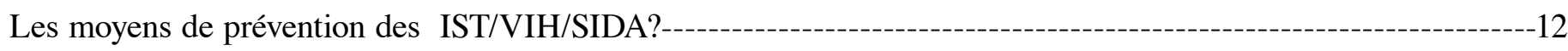

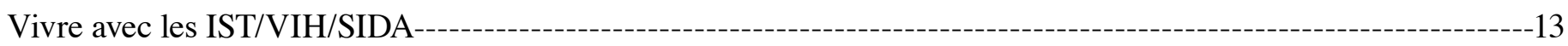




\section{Adolescence et puberté}

J'ai 17 ans, je suis attiré(e) par les garçons (filles), qu'est ce qui m'arrive?

: Tu as 17 ans, c'est l'âge où ton corps se transforme, tu grandis, tu es en train de devenir adulte, il est normal que tu sois attiré(e) par les garçons ou les filles.

J'ai envie de draguer, mais je ne sais pas comment faire?

D : Tu as envie de séduire, mais c'est vrai ce n'est pas facile. Si tu veux draguer, essaie de parler avec la fille, de lui faire comprendre que tu voudrais la connaître et passer du temps avec elle. Mais attention, ne sois pas trop insistant.

\section{Changements physiques}

Q

Mes seins grossissent mais il y'a un qui est plus gros que l'autre, est ce que je suis anormale?

D : Pendant la croissance, cela peut se produire, ce n'est pas alarmant, néanmoins si tu le souhaites tu peux aller rencontrer quelqu'un au centre de conseil ou à un service de santé.

Q

Depuis quelques mois, la nuit je mouille mes draps et au matin j'ai le sexe dur. Comment faire pour que cela cesse? Puis-je le contrôler?

D : Tu es en train de grandir. La nuit tu fais des rêves qui sont très agréables et du sperme sort de ton R pénis. De même le matin tu as des érections c'est tout à fait normal, ce sont tes sens qui s'éveillent. Tu pourras bientôt contrôler cela de mieux en mieux.

\section{Cycle menstruel}

: Qu'est ce qui explique l'irrégularité de mes règles?

D : Toutes les femmes n'ont pas nécessairement des règles régulières, surtout les jeunes filles, mais pour plus d'informations, je te conseille d'aller auprès du centre conseil ou d'une structure de santé pour te faire examiner et te rassurer.

Q

Les règles sont- elles douloureuses?

D: Chez la jeune fille les règles peuvent être douloureuses, s'accompagner de fatigue et de vomissements. En grandissant, ces troubles vont cesser. La prise de calmants vendus en pharmacie apaise rapidement les douleurs.

Q

Qu'est ce qui explique qu'une émotion peut empêcher la venue des règles?

: La vie sexuelle et reproductive des hommes et des femmes est conditionnée par le mental. une émotion forte, un chagrin, une joie, une angoisse peuvent retentir sur nos organes et empêcher leur fonctionnement régulier. 


\section{Changements émotionnels}

: Depuis quelques temps, je suis toujours triste, je reste chez moi, je n'ai plus envie de voir mes amis? Qu'est-ce qui m'arrive?

: C'est vrai souvent à ton âge on est triste ou découragé et parfois sans raison. T'enfermer n'est pas la solution, tu es triste mais il ne faut pas rester seul(e). Tu dois sortir, rencontrer des ami(e)s pour leur parler de ce qui te rend triste, te distraire; il(elle)s sauront sûrement te dire des choses pour te réconforter. Inscris-toi dans un club de sport, de thêatre...

\section{Changements relationnels}

Q

Avec mes parents, c'est difficile. Ils ne comprennent pas ma façon de m'habiller, si je veux aller au bal ils me disent que je suis une prostituée, je pense qu'ils ne m’aiment pas.

D : Lorsqu'on grandit, on veut faire des choses selon son goût et quelques fois il y a des désaccords avec les parents. Il faut parler avec eux pour arriver à se comprendre et pouvoir leur expliquer que ton seul but c'est de voir des amis. Ils ont peur pour toi, ils ont peur que tu tombes enceinte ou qu'il t'arrive quelque chose.

\section{Amour et désir sexuel}

: Comment reconnaître l'amour du désir?

: L'amour est un sentiment qui dure, qui se suffit à lui-même. Le désir est lié à la recherche d'une satisfaction physique, sexuelle. Il peut être superficiel et disparaître une fois l'aboutissement obtenu.

Q

Lorsque mon ami est avec moi, il est toujours en érection, est-il normal?

: Ce que tu dis montre que ton ami est troublé par ta présence et qu'il éprouve du désir pour toi, ce n'est pas anormal entre deux personnes qui sont proches. Le sexe de l'homme devient dur lorsqu'il éprouve du désir pour une personne.

\section{Culture et sexualité}

Q

Que va être la réaction de mes parents si je leur apprends que j’ai eu des rapports sexuels?

: Il se peut qu'ils ne soient pas contents, tu connais nos valeurs. N'as tu pas dans ton entourage, une tante, un oncle auxquels tu pourrais te confier et qui te permettront de dialoguer avec tes parents. Tu peux aussi te rendre au centre conseil ou au poste de santé le plus proche.

\section{Sexualité}

: Comment savoir si je suis séduisante?

: Tu peux le savoir en étant attentive aux comportements des hommes en ta présence, s'ils te font des compliments, s'ils sont gentils: c'est que tu dois être agréable à regarder. Mais ne te laisse pas séduire par ces flatteurs, et préfère la compagnie de personnes plus sérieuses et travailleuses. Par ailleurs, la beauté interne est plus durable et importante que la beauté physique. 
Comment peut on être responsable de sa sexualité, on y peut rien c'est le corps qui parle?

$\mathrm{R}$ : Ton corps te parle et te donne envie d'avoir des rapports sexuels, mais tu dois aussi réfléchir avant d'agir et penser aux conséquences de ce que tu vas faire.

: Est ce qu'il est normal d'avoir une activité sexuelle?

R : Nous avons tous une sexualité, notre corps et notre tête ressentent des choses agréables dans certaines situations ou en présence de certaines personnes. Nous devons choisir le moment pendant lequel et aussi la personne avec laquelle nous aurons des relations approfondies.

Q: со Comment savoir si ce soir je vais avoir un rapport avec ma copine?

Avant d'avoir un rapport tu dois bien réfléchir si c'est ce que tu veux vraiment et connaître son avis sur la question. Ce n'est pas facile ; ne te précipite pas

$\mathrm{Q}_{\mathrm{R}}^{s}$ J'ai un copain, je l'aime, il dit que si je l'aime, je dois avoir des rapports sexuels avec lui?

D : Avoir un rapport sexuel est un acte qui se fait à deux et peut avoir des conséquences (grossesse etc.... problèmes avec les parents). Parle avec ton copain, dis-lui que tu préfères attendre, tu n'es pas prête. S'il t'aime, il respectera ta décision.

Q Tous les jeunes que je connais ont des rapports avec des filles ou des femmes. Pourquoi pas moi? Estce que moi je ne devrais pas?

: Les jeunes que tu connais te disent qu'ils ont des rapports, mais souvent, ce n'est pas la vérité. Ils le disent pour se vanter, pour "être dans le coup". Cela ne doit pas te forcer dans tes choix. Il faut respecter les valeurs de notre pays et attendre le mariage,ou en tout cas d'être mûr(e) et prêt(e) pour les rapports sexuels.

Q La taille de mon pénis n'est pas grande, pourrais je avoir des rapports avec une femme et la satisfaire?

: La taille du pénis n'est pas importante pour le rapport et le plaisir. Le vagin de la femme est Célastique et il s'adapte à la taille du pénis du partenaire. Le plaisir dépend de la façon dont l'homme sera attentif et tendre avec sa partenaire.

\section{La santé sexuelle}

Quels sont les problèmes de santé sexuelle?

$\mathbf{R}$ : Tous les troubles du fonctionnement des appareils génitaux de l'homme et de la femme. 
Pourquoi faut-il aller régulièrement se faire consulter?

: Pour prévenir des problèmes de prostate, de cancer du col de l'utérus, de descente d'organes... Pour éviter de contacter une IST/VIH/SIDA ou une grossesse, ou encore en cas de stérilité.

: Quand faut-il aller se faire consulter?

: En cas de douleur, grosseur, bouton, plaie, écoulement, odeur forte, saignement au niveau des organes génitaux.

\section{La masturbation}

Q: Qurdur

Quelqu'un qui se masturbe beaucoup devient un obsédé sexuel

: La masturbation n'entraîne pas l'obsession sexuelle. C'est une pratique courante chez l'individu qui aborde la puberté et qui a tendance à s'estomper avec l'âge.

Q

Est-ce que la masturbation distrait l'individu de l'école ou de son travail?

D : Non, cependant, comme le rappelle l'adage "tout excès est nuisible".Si la masturbation permet de temps en temps de se libérer de la tension sexuelle, elle ne doit pas être pratiquée de façon excessive.

: La masturbation empêche t-elle d'avoir une relation sexuelle avec une personne de sexe opposé?

$\mathbf{R}$ : Non la masturbation n'empêche en rien un individu d'avoir un rapport sexuel avec une autre personne.

Q

: Est-ce que seuls les hommes se masturbent?

R: Aussi bien les hommes que les femmes pratiquent la masturbation.

Q

Est-ce que la masturbation rend fou?

R: La masturbation en elle-même n'a aucune conséquence sur la santé mentale.

Q

: Est-ce que la masturbation est dangereuse?

R: Il ne peut pas être dangereux de se masturber, il n'y a aucun risque physique. 
: Est-ce que je ne vais pas trop aimer cela?

R : Il s'agit simplement de faire baisser la tension sexuelle en l'absence d'un partenaire.

\section{Troubles de l'érection}

Depuis quelques jours, je ne parviens pas à avoir une érection, pourtant j'aime beaucoup ma femme. Qu'est ce qui se passe?

: Tu as raison de prendre au sérieux ce genre de situation. Ce n'est pas très grave, l'homme n'est pas une machine. Peut-être es tu fatigué ou as tu des soucis et cela agit sur ton corps. Pour en savoir plus, je te propose d'aller rencontrer quelqu'un au centre conseil ou à la structure de santé.

\section{La sexualité à moindre risque}

Est-ce qu'on peut tomber enceinte pendant le premier rapport?

: Oui on peut tomber enceinte pendant le premier rapport, à partir du moment où le sperme est entré dans le vagin, il peut y avoir une grossesse.

Q

Est-ce que je peux tomber enceinte sans pénétration?

: Oui tu peux tomber enceinte sans pénétration, si ton partenaire éjacule près de ton sexe, il est possible que son sperme puisse pénétrer dans ton vagin.

: Est-ce qu'une fille peut tomber enceinte tout en restant vierge?

: L'hymen n'est pas étanche, il comporte des trous par lesquels les règles s'écoulent; les spermatozoïdes peuvent passer par ces trous et causer une grossesse.

: A quel moment je ne risque pas d'enceinter une fille?

: À certain moment du mois, la femme est plus féconde que d'autres. Environ 14 jours après le début des règles, la femme risque, si elle a un rapport sexuel non protégé de débuter une grossesse. Il faut donc s'abstenir. Mais parfois la femme ne voit pas ses règles régulièrement; il lui est donc difficile de prévoir car elle peut tomber enceinte à tout moment. C'est pourquoi il faut toujours utiliser un préservatif.

Je veux avoir des rapports avec mon ami mais j'ai peur de tomber enceinte?

: Il existe des méthodes pour ne pas tomber enceinte, la pilule, l'injectable ou encore le préservatif qui reste le meilleur moyen de se protéger contre les grossesses et les IST/SIDA... Vas te renseigner au centre conseil ou au centre de santé où on te donnera plus d'informations. 
: Le rapport sexuel est il douloureux et violent?

$\mathrm{R}$ : Un rapport sexuel ne doit pas être un acte violent. L'homme doit être très doux et attentif à sa partenaire pour éveiller en elle l'envie et le désir. Il ne doit pas faire preuve d'égoïsme dans la recherche de son plaisir.

Q

Est-ce qu'une femme peut refuser un rapport sexuel à son ami ou son mari?

D : Dans certains cas, la femme n'éprouve pas le désir, l'homme doit respecter la volonté de la femme. La femme a parfois des règles douloureuses ou elle est fatiguée par les tâches quotidiennes ou elle a des soucis.

Q

Comment je peux être sûr(e) d'avoir des rapports sexuels à moindre risque?

: Si tu t'abstiens de rapport avec pénétration ou si tu utilises le préservatif, tu pratiques la sexualité à moindre risque.

\section{Le préservatif}

Est-ce que le préservatif diminue le plaisir?

: Le siège du plaisir sexuel se situe au niveau du cerveau. Physiquement, le préservatif n'est pas un facteur de diminution ou d'inhibition du plaisir sexuel.

Q

Est-ce que le VIH peut traverser le préservatif?

R : Un préservatif en cours de validité et qui est bien conservé ne peut être traversé par le VIH.

Q

Faut-il doubler le préservatif pour être bien protégé?

: Le préservatif est fabriqué dans le respect des normes de qualités fiables. Il n'est pas nécessaire de le doubler, sinon, ils risquent de se déchirer.

Q

Est-ce que le préservatif diminue la virilité?

R: Il n'y a pas de lien entre l'utilisation du préservatif et l'augmentation ou non de la virilité.

Q

Le VIH est-il contenu dans certains préservatifs?

: Le VIH est contenu dans le sang, le sperme, les sécrétions vaginales et le lait maternel. Le préservatif neuf est totalement stérile. 
: Est-il vrai que le préservatif se déchire facilement?

: Le préservatif est fabriqué avec du latex qui est une matière résistante. Il est testé avant d'être mis sur le marché. Cependant, il faut prendre des précautions quant à sa conservation et sa manipulation. Eviter de l'exposer au soleil, de le garder dans la poche arrière d'un pantalon serré, de l'ouvrir avec les dents, les ongles.

Q L'utilisation du préservatif traduit-elle un manque de confiance?

D : L'utilisation du préservatif est plutôt un acte de responsabilité. Non seulement c'est un moyen de protection contre les IST, mais aussi, il nous évite des grossesses non désirées sources de beaucoup de problèmes.

Le préservatif est-il réutilisable?

: Le préservatif s'utilise strictement une seule fois.

Maltraitance - violence - abus sexuels

Q J'ai 13 ans. J'ai été victime d'un viol. C'était lors d'un séjour chez mon oncle, pendant les vacances. J'étais entrain de ranger la chambre du fond, lorsqu'un cousin est entré dans la pièce et m'a forcé à avoir un rapport sexuel avec lui. Dois-je en parler? Depuis j'ai d'intenses douleurs au ventre.

: Tu dois en parler à ta mère ou ta tante. C'est elle qui pourra te conduire au service de santé afin de C te faire consulter. Tu dois le dire car tu vas encore rencontrer ce cousin et il ne faut pas que cela se reproduise. La loi punit cet acte et tu peux porter plainte contre cette personne.

\section{L'homosexualité}

Q

: Je m'appelle Abdou, J'ai 16 ans, j'aime beaucoup être en compagnie de Souleymane. L'autre jour j'ai dormi avec lui et au réveil j'avais mouillé mon pantalon, est ce que je suis un homosexuel?

D : Il y a de l'homosexualité en chacun de nous, il ne faut pas t'en affoler. Il ne faut pas croire que tu est pour autant homosexuel. Il arrive que l'on éprouve des émois physiques devant quelqu'un du même sexe. Ce ne sont que des moments. Tu as dû dans ton sommeil faire un bon rêve ce qui est fréquent quand on est jeune.

\section{Les grossesses indésirées}

: Je suis enceinte, j'ai 16 ans ai-je le droit d'avorter?

: L'avortement est interdit au Sénégal et puni par la loi. Tu cours de graves risques pour ta santé en ayant recours à un avortement. Avoir un enfant à ton âge est difficile, va au centre conseil ou au centre de santé, là-bas des personnes t'attendent pour te permettre de te préparer à cette naissance. 


\section{La parenté responsable}

Comment savoir si je suis prêt(e) au mariage et à être parent?

D : Tu as bien réfléchi(e) avec ton partenaire, si vous avez les mêmes idées sur la vie, le rôle et la place de chacun, si vous avez des projets ensemble. Tu peux te rendre au centre conseil ou au centre de santé et rencontrer un des spécialistes pour en parler.

\section{La contraception}

Pourquoi est-ce que je devrais utiliser la contraception?

: La planification familiale te permet de mieux planifier ta vie, si tu ne peux pas t'abstenir elle te permet d'éviter les grossesses.

Q

Qu'est ce qu'une méthode de planification familiale?

: C'est un moyen qui permet au couple d'espacer les naissances dans le but de protéger la santé de la mère et de l'enfant et d'éviter des grossesses tardives, rapprochées et indésirées.

Q

Est ce que les méthodes de planification familiale sont dangereuses?

R: Non, ces méthodes ne présentent aucun danger si les conditions d'utilisation sont respectées.

Q

Est ce que l'injectable provoque des saignements?

: L'injectable peut provoquer de petits saignements en dehors de la période des règles sans gravité. Si tu veux être rassurée va au centre de planification familiale le plus proche.

Q

Est-ce que l'islam est pour les méthodes modernes de planification familiale?

R: L'Islam reconnaît la possibilité de recourir aux méthodes de planification familiale afin de préserver la santé de la femme et de l'enfant.

J'ai entendu dire que les méthodes de planification familiale rendent la femme stérile. J'ai peur d'en utiliser une.

: Les méthodes de planification familiale empêchent une grossesse mais ne rendent pas stérile. Seule la stérilisation féminine également appelée ligature des trompes et la vasectomie sont des méthodes définitives. Les autres méthodes sont des méthodes temporaires pour un moment donné et peuvent être arrêtées dés que la femme le souhaite. Pour en savoir plus, rends-toi au centre de planification familiale. 
: Je prends la pilule depuis deux ans. On m'a dit que le produit s'accumule dans le ventre et peut me rendre malade. Dois-je abandonner cette méthode?

$\mathrm{R}$ : La pilule est un médicament comme un autre. Les hormones qu'elle contient se dissolvent dans le Corps humain, elles ne s'accumulent pas. Tu es libre de changer de méthode. Pour en savoir plus, rends-toi dans un centre de santé.

\section{Les Infections Sexuellement Transmissibles (IST)}

Q

Est ce que l'on peut attraper une IST lorsqu'on est jeune et non marié?

: Les IST s'attrapent au cours des relations sexuelles. Si tu es sexuellement actif, tu peux en avoir une.

Quelles sont les IST les plus courantes au Sénégal?

: La gonorrhée, le chancre mou, la syphilis, La chlamydia, l'herpès

\section{Modes de transmission des IST}

Q

Le contact bouche sexe peut-il transmettre les IST?

: Les contacts de la bouche avec le sexe peuvent transmettre les IST surtout s’il y a des lésions dans la bouche ou sur le sexe.

Q

Le baiser peut-il transmettre les IST?

: Le baiser présente un risque très faible mais théoriquement possible en particulier pour l'hépatite $\mathrm{B}$ et l'Herpès des lèvres ou des gencives quand il $\mathrm{y}$ a des lésions.

Q J'ai entendu qu'on peut attraper une IST en mangeant du poisson à la chair noire. Est -ce vrai?

: Il y a beaucoup de choses que l'on dit et qui ne sont pas exactes. Les IST se transmettent par les relations sexuelles avec une personne infectée, cela ne se transmet pas par la nourriture.

\section{Signes ou symptômes des IST chez la femme et chez l'homme}

: J'ai mal au sexe et j'ai des plaies autour de mon sexe...que dois je faire?

: Souvent, des plaies autour des organes génitaux indiquent une infection sexuellement transmissible. Je te suggère d'aller immédiatement au centre de santé te faire traiter. Les IST peuvent avoir des conséquences graves si elles ne sont pas soignées immédiatement. Suis le traitement d'un docteur, ou d'un agent de santé et n'achète pas les médicaments au marché. Tu devrais également informer ta partenaire si tu as une IST afin que celle-ci puisse se protéger, se faire traiter si elle est atteinte et ne pas te retransmettre la maladie. 


\section{Que faire si on a une IST?}

Je crois que j'ai attrapé une maladie lors d'un rapport sexuel?

: Va au centre de santé. Surtout ne te traite pas toi-même et préviens ta partenaire pour qu'elle se fasse traiter.

Q

peut-on avoir plusieurs IST en même temps?

: C'est très fréquent surtout chez les personnes qui ont des partenaires multiples. Une IST peut en cacher une autre dont les manifestations sont plus discrètes. La présence d'une IST peut favoriser la venue d'une autre infection par la présence de plaies. C'est le cas du SIDA.

: Peut on avoir la même IST plusieurs fois?

: Oui en dehors de l'hépatite B, les autres IST peuvent s'attraper plusieurs fois si on ne se protége pas à chaque rapport sexuel avec le préservatif.

\section{Conséquences des IST}

Q: Qu'sice

Qu'est ce que je risque si j'attrape une IST?

: Si tu attrapes une IST tu mets en danger ta santé. Tu vas rendre malade toutes les personnes avec lesquelles tu auras des rapports sexuels. Tu peux devenir stérile, avoir des enfants qui seront malades à la naissance. Ne prends pas ce risque. Pour en savoir plus, va rencontrer un spécialiste au centre de santé, il t'en dira plus.

\section{Le VIH/SIDA}

: D'où vient le SIDA

: On ne connaît pas l'origine du SIDA. Il est présent dans le monde entier et il atteint tout le monde. C'est un produit de la nature et non quelque chose créée par l'homme.

Q

: Est-ce que le moustique donne le SIDA?

R: Le moustique ne transmet pas le SIDA, s'il le faisait, nous l'aurions tous.

Q

Peut-on avoir le SIDA par la salive en embrassant?

R: On ne connaît aucun cas de personnes infectées par le SIDA à la suite d'un baiser. 
: Combien de temps le VIH peut-il vivre à l'extérieur du corps?

: Le virus est très fragile et ne peut vivre hors du corps très longtemps. Le VIH peut être facilement tué en lavant la partie exposée à l'eau et au savon, en ajoutant une mesure d'eau de Javel pour dix mesures d'eau.

: La masturbation réciproque transmet-elle le SIDA?

: La masturbation réciproque est sans risque sauf s'il y a contact d'une légion génitale ou de sperme avec une plaie ouverte de la main.

\section{Le test de dépistage}

J'ai peur que tout le monde sache que je veux passer un test de dépistage.

R: Sois sans crainte, le secret sera gardé par le personnel médical.

Q : Faut-il attendre au minimum 3 mois après un risque de contamination afin de pouvoir faire un test de dépistage du SIDA (test VIH)?

D : Le test des anticorps anti-VIH (Elisa) est une méthode permettant d'apporter indirectement la C preuve de l'infection par le VIH. Il met en évidence les anticorps qui se forment dans le sang d'une personne contaminée généralement 1 mois après l'infection. Un test de dépistage des anticorps anti-VIH n'est certain que si le dernier risque pris (rapport sexuel, injection de drogue...) remonte à 3 MOIS au moins avant le test. Mais il existe maintenant d'autres tests qui, avant l'apparition des anticorps, peuvent mettre en évidence la présence du VIH. Ces tests peuvent être pratiqués 10 ou 15 jours après le risque de contamination.

: Combien de temps faut-il entre une prise de risque et le test pour que le résultat de celui-ci soit certain?

D : La certitude de la non-infection avec un test (Elisa) est de 3 mois. Cependant dans de nombreux cas en raison de la sensibilité des tests actuels, il est possible d'affirmer plus tôt le résultat. Cela ne veut pas dire qu'il faut attendre 3 mois sans rien faire: aujourd'hui après une exposition au risque VIH il ne faut plus attendre, mais consulter un médecin en urgence car un traitement après exposition peut-être indiqué. Il doit débuter au mieux dans les toutes premières heures et, en tout cas, dans les 48 heures qui suivent l'exposition au risque. Actuellement, le protocole de dépistage sérologique et virologique permet de rechercher directement les traces du VIH entre 10 et 15 jours après la contamination.

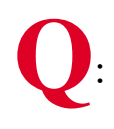

Que signifie un test de dépistage négatif?

D: Que le sang de la personne ne contenait pas de VIH 3 mois avant le test. Les anticorps antiR VIH apparaissent dans un délai de 2 à 4 semaines en moyenne après la contamination. Le test de dépistage des anticorps est donc négatif au tout début de l'infection, même si la personne est contaminée. D'autres examens peuvent cependant permettre de diagnostiquer l'infection avant l'apparition des anticorps... En pratique, être "séronégatif" signifie que la personne n'était pas contaminée par le VIH 3 mois avant de faire le test. Si cette personne n'a eu depuis que des rapports protégés (avec préservatif), et pas de risque de contamination par voie sanguine, elle est sûre de ne pas être contaminée par le VIH, sinon, elle devra refaire un test ( 3 mois après le dernier rapport non protégé) pour avoir une réponse certaine. Un résultat négatif ne constitue pas pour autant une protection définitive contre la maladie. Etre séronégatif à un certain moment ne signifie pas que l'on est pour toujours hors de danger. Les règles de prudence continuent de s'imposer pour éviter la contamination. 


\section{Les moyens de prévention des IST/VIH/SIDA?}

Q

J'ai un rapport sexuel et j'ai peur d'avoir attrapé une IST ou le SIDA, que dois-je faire?

D : Tu as raison de t'inquiéter de ta santé. Va au centre conseil adolescent ou au centre de planning familial, tu rencontreras une sage-femme qui saura te renseigner, et éventuellement te traiter.

Q

Je ne sais pas où acheter des préservatifs.

: tu peux acheter des préservatifs dans les pharmacies, dans certaines boutiques, dans les services de santé; surtout ne les achète pas sur les marchés parce qu ils ne sont pas de bonne qualité.

Q

Si je me balade avec un préservatif, ma copine va croire que je ne suis pas un garçon sérieux.

D: Ce n'est pas parce que tu as un préservatif sur toi que tu es prêt à "draguer" toutes les filles. Explique-lui que c'est parce que tu la respectes et que tu tiens à rester en bonne santé et à ne pas la rendre malade que tu as des préservatifs sur toi.

Q

Cela me gêne d'aller en acheter.

: Tout le monde devrait en acheter, la sexualité n'est pas sans danger. Va en acheter dans un autre quartier que le tien, là où personne ne te connaît. Fais ce qu'il faut pour protéger ta santé.

Q:

Faire l'amour avec un préservatif n'est pas aussi bien!

D : C'est dans la tête que cela se passe. Certains hommes trouvent que grâce au préservatif, ils éjaculent moins rapidement. En plus, si tu attrapes une IST, les rapports sexuels peuvent être plus douloureux.

Q

Un vrai homme n'utilise pas le préservatif!

: Un vrai homme prend soin de son corps et de sa santé. C'est simplement la preuve que tu es intelligent et responsable et que tu te soucies de la santé de l'autre.

: Ma religion m'empêche d'utiliser un préservatif, c'est un pêché.

R: Il est important d'être en accord avec ses choix, avec ses croyances. Demande conseil à tes amis, ton Imam, ton Prêtre, ils t'aideront à y voir plus clair. Si tu souhaites que ton partenaire te préserve d'une infection, alors tu dois t'engager à ne pas la contaminer. 
Une femme séropositive devrait-elle éviter d'être enceinte?

: C'est un choix individuel qui engage chacun. Une femme infectée a un risque sur trois de donner naissance à un enfant infecté. Si l'enfant n'a pas été infecté au cours de la grossesse et de l'accouchement, il risque d'être infecté pendant l'allaitement. Est-ce que cet enfant aura quelqu'un qui puisse s'occuper de lui s'il venait à perdre sa mère? Chacun doit peser ses décisions en étant informé des risques.

Q

Je viens d'apprendre que j’ai le virus VIH dans mon corps est-ce que je vais mourir demain?

: Tu as le virus dans ton corps mais tu n'a pas encore commencé à développer la maladie, tu as encore de nombreuses années à vivre si tu respectes certaines précautions: porter un préservatif à chaque rapport, surveiller ta santé en te rendant régulièrement en consultation, bien manger et ne pas faire d'abus.

$\mathbf{Q}_{\mathrm{R}}^{\text {sit }}$ Si je suis séropositif, est ce que je peux quand même avoir des rapports sexuels?

: Bien sûr, mais il n'y a pas de sexualité sûr à 100\%. Tu dois avertir ton partenaire de ta séropositivité de façon à prendre ensemble une décision. Pratiquer la sexualité à moindre risque en mettant un préservatif du début à la fin du rapport.

\section{Q}

Est-ce qu'il existe des médicaments pour guérir le SIDA?

: Il n'y a pas actuellement de moyen pour guérir du SIDA. Mais il y a des traitements pour soigner toutes les maladies qui viennent avec l'infection. Des recherches sont en cours dans les laboratoires, et bientôt le traitement sera au point. Il y a des associations de médicaments : les anti rétro viraux (ARV). Le traitement est long et il permet une rémission de l'infection mais le malade n'est pas guéri. Il doit continuer à prendre le traitement toute sa vie et c'est très contraignant. Au Sénégal, le traitement est gratuit. 


\section{Crédits photos et images}

Choices, A Guide for Young People, Gill Gordon. 1999

Preparing for the VIBES in the World of Sexuality, Joseph E. Robinson/Ashe. 1999

Population Council

www.medisite.fr 